
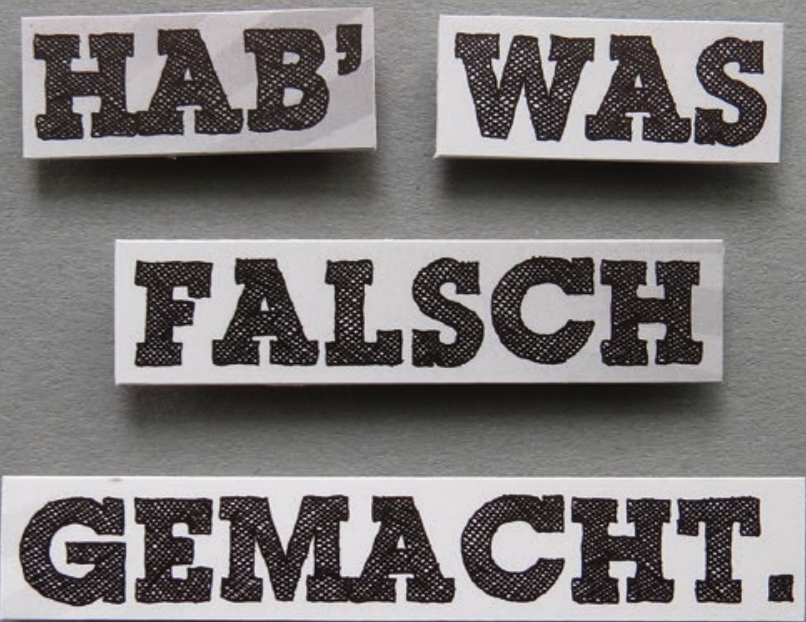

\title{
Verschweigen hilft nicht
}

Wer kritisch kranke Menschen pflegt, darf sich keine Fehler leisten. Diese Null-Fehler-Mentalität wirkt von allen Seiten auf die Intensivpflege ein - Patienten, Angehörige, Rechtsprechung, Vorgesetzte und Kollegen setzen fehlerfreies Arbeiten voraus. Von der erwünschten Null-Fehler-Realität sind wir dagegen weit entfernt: Fehler in der Intensivpflege gehören zum Alltag. - Autor Christian Bibo zeigt Wege zu einer besseren Fehlerkultur. Seine Arbeit belegte den

\section{Platz beim Thieme intensiv-Pflegepreis 2013.}

Im Sinne der Patientensicherheit ist es unabdingbar, sich mit Fehlern offen auseinanderzusetzen - was oft schwerfällt. Dieser Beitrag geht der Frage nach, wie sich im Team der Intensivstation eine förderliche Fehlerkultur etablieren lässt. Zunächst werden Häufigkeit, Begriff, Entstehung von Fehlern sowie ihre Untersuchung und Behandlung erörtert. Anschließend wird ein Beispiel aktueller Fehlerkultur in der Intensivpflege analysiert und mit dem Ideal verglichen. Hieraus werden notwendige Veränderungen identifiziert und Wege zu deren Umsetzung vorgestellt. Ein Fazit schließt den Beitrag ab.

\section{Fehlerhäufigkeit in der Intensivpflege}

Wie häufig in der Intensivpflege Fehler geschehen, ist unmöglich anzugeben. Es sei hier auf das oft angeführte Eisbergmodell der Unfälle verwiesen: Kritische Ereignis- se ( $\rightarrow$ Glossar Patientensicherheit) werden wahrgenommen, wenn sie zu großem Schaden führen: Sie bilden die „Spitze des Eisbergs“. Die vielfach größere Zahl von kleineren oder Beinahe-Schäden bleibt, wie der Großteil eines Eisbergs, unter Wasser.

Um eine grobe Vorstellung der Fehlerhäufigkeit zu erhalten, sollen hier zwei multinationale Beobachtungsstudien vorgestellt werden: Die SEE-Studie von Valentin et al. untersuchte Häufigkeit und beitragende Faktoren kritischer Ereignisse auf weltweit 205 Intensivstationen. Es wurden nur solche Ereignisse erfasst, die durch aktive Handlungen herbeigeführt wurden. Als repräsentative Kategorien wurden die Medikation, Zugänge und Drainagen, Geräteausfälle, künstliche Atemwege und das Alarmmanagement gewählt. Die ermittelte Häufigkeit kritischer Ereignisse entsprach 38,8 pro 100 Patiententage, wobei die Autoren auf- grund der Datenerfassung per Selbstbericht eine Dunkelziffer vermuten. ${ }^{2}$

Eine zweite Studie ähnlichen Designs von Valentin et al. untersuchte Fehler bei der parenteralen Applikation von Medikamenten auf weltweit 113 Intensivstationen. Wieder wurden Daten durch Selbstbericht gesammelt, diesmal auch Fehler durch Unterlassung und entstandene Schäden erfasst. Es wurden 74,5 Fehler pro 100 Patiententage ermittelt. Von den 861 berichteten Fehlern führten 15 zu bleibendem Schaden oder zum Tod: ${ }^{3}$ ein schwerer Schaden pro 57 kritische Ereignisse.

Amalberti stellte 2001 eine Einteilung soziotechnischer Systeme in riskante, regulierte und ultrasichere Systeme vor. Systeme, in denen aus 1.000 kritischen Ereignissen mehr als ein schwerer Unfall entsteht, gelten als riskant. ${ }^{4}$ Diesen Systemen, zu denen Amalberti beispielsweise Klettersport oder Bungee-Jumping zählt, wären mit 
obigem Ergebnis Intensivstationen zuzuordnen. Grund genug, sich mit dem Begriff des Fehlers näher zu beschäftigen.

\section{Was ist ein Fehler?}

Die Definition von Rall et al. ( $\rightarrow$ Definition: Fehler) bezeichnet Fehler als „negative Reaktion" und zielt damit eher auf Fehlerfolgen: Nicht jeder Fehler verursacht negative Reaktionen. Wichtige Feststellungen sind, dass auch Nicht-Handeln ein Fehler sein kann und dass Fehler bewusst und unbewusst entstehen können.

Beim Vergleich dieser Fehlerdefinition mit der des Aktionsbündnisses Patientensicherheit fällt auf, dass letzteres den Fehler von seinen Auswirkungen her differenziert. Etwas weniger scharf ist die Beschreibung der Fehlerentstehung: Eine vom Plan abweichende Handlung oder Unterlassung kann durchaus richtig sein, wenn sie bewusst und begründet erfolgt (Beispiel: Es wird erkannt, dass der Plan auf eine veränderte Situation nicht mehr sicher anwendbar ist). Falsche oder fehlende Planung sind unzweifelhaft mögliche Fehlerursachen.

Die Fehlerdefinition des Erziehungswissenschaftlers Martin Weingardt ist transdisziplinär ausgelegt und daher allgemein gehalten. Für Verständnis und Beurteilung von Fehlern sind folgende Punkte von Bedeutung:

- Subjektivität: Was ein Fehler ist und was nicht, liegt im Auge des Betrachters.

- Ohne Alternative kein Fehler: Unausweichliche Handlungen oder Unterlassungen können keine Fehler darstellen.

- Kontext: Die Situation um den mutmaßlichen Fehler muss bei der Beurteilung berücksichtigt werden.
- Interesse: Welche Ziele verfolgte der Handelnde? War sein Handeln, gemessen an diesen Zielen, angebracht? Zielkonflikte beachten: Eine für ein Ziel angemessene Handlung kann hinsichtlich eines anderen Ziels nachteilig sein!

\section{Die Entstehung von Fehlern}

Warum und auf welche Weise sich Fehler in unser Tun einschleichen, soll anhand der Arbeiten des Psychologen James Reason erläutert werden. Dabei sind vorab folgende Grundgedanken von Bedeutung:

- Unfälle sind multifaktoriell: In komplexen Systemen wie etwa Intensivpflege und -therapie haben kritische Ereignisse fast immer mehr als nur eine Ursache. Reason spricht von einer „Fehlerkette“ aus vielen zusammenwirkenden Faktoren. $^{7}$

- Beitragende Unfallursachen können örtlich und zeitlich weit vom Geschehen entfernt sein. Solche Faktoren bezeichnet Reason als „latente Bedingungen“. Beispielhaft können dies Umwelteinflüsse (Stromausfall) oder Entscheidungen des Managements (Arbeitsverdichtung) sein. Sie werden oft erst dann als Gefahren erkannt, wenn sie gemeinsam mit lokalen Auslösern zum Zwischenfall führen. ${ }^{7}$

- Das, was wir als Fehler kennen, nennt Reason „aktive Fehler“ oder „unsichere Handlungen“. Er sieht in ihnen keine Ursachen, sondern Konsequenzen der menschlichen Natur, vor allem aber der oben genannten latenten Bedingungen. ${ }^{7}$ Unsichere Handlungen werden in $\rightarrow$ Tab. 1 klassifiziert.

\section{Definition: Fehler}

Rall, et al. 2001:

„Nicht beabsichtigte, oft auch nicht erwartete negative Reaktion auf eine bewusst oder unbewusst ausgeführte, oder unterlassene Maßnahme.“5

Aktionsbündnis Patientensicherheit 2007:

„Eine Handlung oder ein Unterlassen, bei dem eine Abweichung vom Plan, ein falscher Plan oder kein Plan vorliegt. Ob daraus ein Schaden entsteht, ist für die Definition des Fehlers irrelevant."1

Weingardt 2004:

„Als Fehler bezeichnet ein Subjekt angesichts einer Alternative jene Variante, die von ihm - bezogen auf einen damit korrelierenden Kontext und ein spezifisches Interesse als so ungünstig beurteilt wird, dass sie unerwünscht erscheint." 6

\section{Wie reagieren auf welchen Fehler?}

Fehler bei der Handlungsausführung geschehen unbewusst. Mit „besser aufpassen“ ist ihnen nicht beizukommen, ebenso wenig mit Sanktionen wie arbeits- oder strafrechtlichen Maßnahmen - sie sind kein individuelles Problem der persönlichen Eignung. ${ }^{7}$ Bei standardisierten Abläufen kann die Anwendung gut lesbarer Checklisten helfen, beim Vorbereiten von Medikamenten das laute Vorsagen von Medikament und Dosierung während der Tätigkeit. Am wichtigsten ist jedoch die Gestaltung von Umfeld und Arbeitsbedingungen:

- Reduktion von Ablenkung: Lärm, parallel zu erfüllende Aufgaben, Zeitdruck

- Erleichterung der Aufmerksamkeitslenkung: Design von Geräten und Arbeitsplätzen, ausreichende Beleuchtung

- Sicherheitsbarrieren: „intelligente" Spritzenpumpen mit Dosisgrenzen, Spritzen für enterale Nahrung, die nicht auf Gefäßzugänge passen, VierAugen-Prinzip

Fehler der Handlungsplanung lassen sich am leichtesten durch das Vier-Augen-Prinzip und gegenseitige Beratung bekämpfen. Dem Einzelnen kann es helfen, Situationsanalyse und Entscheidungsfindung bewusst schrittweise zu durchlaufen. Hierzu ist die FORDEC-Methode ( $\rightarrow$ Tab. 2 ) dienlich, die vom Deutschen Zentrum für Luftund Raumfahrt für das Pilotentraining entwickelt wurde.

\section{- Glossar Patientensicherheit ${ }^{1}$}

Patientensicherheit

Abwesenheit unerwünschter Ereignisse

Unerwünschtes Ereignis (adverse event) Ein schädliches Vorkommnis, das eher auf der Behandlung denn auf der Erkrankung beruht

Vermeidbares unerwünschtes Ereignis Ein unerwünschtes Ereignis, das vermeidbar ist

\section{Kritisches Ereignis (critical incident)}

Ein Ereignis, das zu einem unerwünschten Ereignis führen könnte oder dessen Wahrscheinlichkeit deutlich erhöht

Beinahe-Schaden (near miss)

Ein Fehler ohne Schaden, der zu einem Schaden hätte führen können 


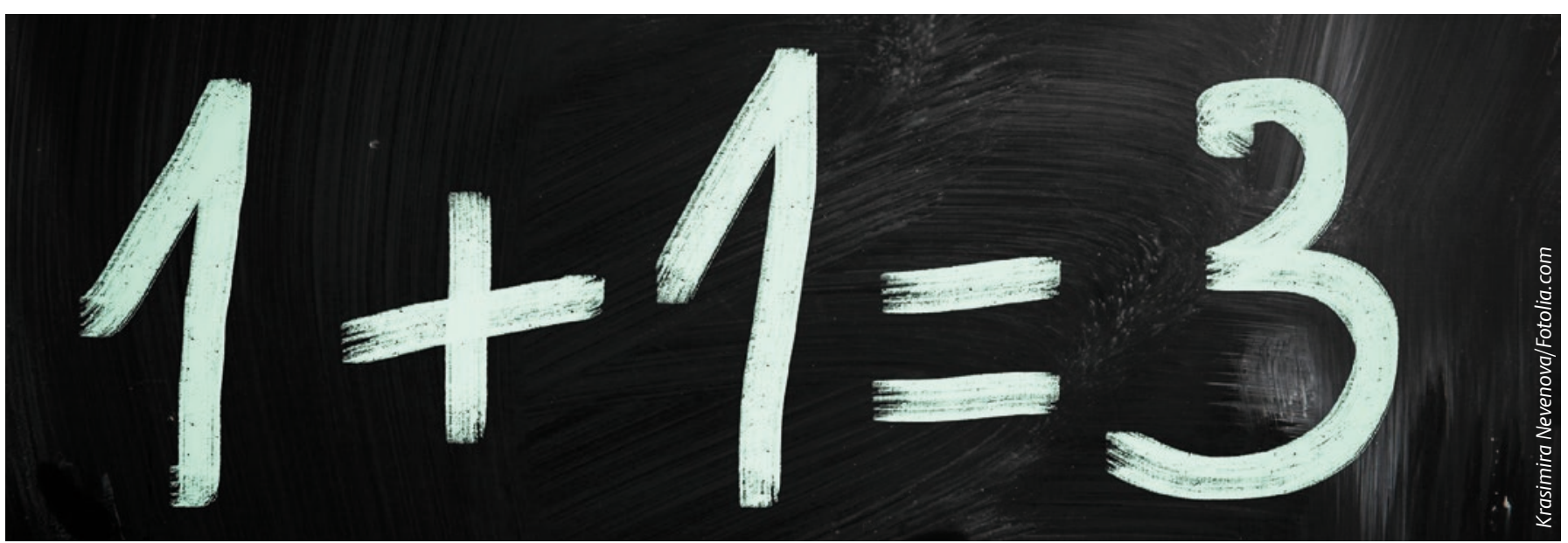

Tab. 1 Klassifizierung unsicherer Handlungen ${ }^{8}$

\begin{tabular}{|c|c|c|}
\hline Entstehungsebene & Typ & Beispiele \\
\hline \multirow[t]{2}{*}{ Handlungsausführung } & Aufmerksamkeitsfehler & $\begin{array}{l}\text { - Zahl falsch ablesen } \\
\text { - Falsche Taste drücken }\end{array}$ \\
\hline & Gedächtnisfehler & $\begin{array}{l}\text { - Arbeitsschritt überspringen } \\
\text { - Vergessen, was man vorhatte }\end{array}$ \\
\hline \multirow[t]{2}{*}{ Handlungsplanung } & $\begin{array}{l}\text { Regel- oder wissens- } \\
\text { basierte Fehler }\end{array}$ & $\begin{array}{l}\text { - Unwissenheit } \\
\text { - Situation falsch interpretieren } \\
\text { - Falscher Plan }\end{array}$ \\
\hline & Verstöße & $\begin{array}{l}\text { - Sabotage } \\
\text { - Handeln nach anderen als den } \\
\text { vorgegebenen Zielen }\end{array}$ \\
\hline
\end{tabular}

Tab. 2 FORDEC-Methode

\begin{tabular}{|l|l|}
\hline Facts & Welche Situation besteht? \\
\hline Options & Was sind die Handlungsoptionen? \\
\hline Risks/Benefits & Pro und Kontra der Optionen \\
\hline Decision & Entscheidung für ein Vorgehen \\
\hline Execution & Ausführung des Plans \\
\hline Check & Evaluation: Ziel erreicht? \\
\hline
\end{tabular}

Tab. 3 Fragen der Fehleranalyse

\section{Personenzentrierte Analyse}

- Welcher Schaden ist eingetreten?

- Wer hat den (!) dazu führenden Fehler gemacht?

- Wer bringt den Schaden wieder in Ordnung?

- Wie ist mit dem Schuldigen zu verfahren?

\section{Systematische Analyse}

- Was wurde entschieden, getan, unterlassen?

- Mit welchem Ziel?

- Unter welchen Bedingungen und Umgebungsfaktoren?

- Gab es Handlungsalternativen?

- Welches Bild der Situation hatten die Akteure?

- Was hat die Akteure zu den fraglichen Entscheidungen/Handlungen bewogen?

- Was kann getan werden, um das Geschehene in Zukunft zu vermeiden?
Verstöße sind keine Fehler im eigentlichen Sinne - die Handlung verfolgt bewusst ein anderes als das vorgegebene Ziel. Sie sind die einzigen der unsicheren Handlungen, auf die mit Sanktionen reagiert werden sollte. Werden Verstöße nicht sanktioniert oder zeigen sie sogar Erfolge, entwickeln sie sich zu Gewohnheiten, sogenannten Routineverstößen ${ }^{8}$ (Beispiel: Missachten der Einwirkzeit bei Desinfektionsmaßnahmen aufgrund von Zeitdruck). Die Sanktionierung als einziges Mittel ist jedoch unzureichend; gleichzeitig muss untersucht werden, ob äußere Einflüsse oder Arbeitsbedingungen zu solchen Verstößen motivieren.

\section{Analyse von Fehlern}

Nach üblichem Verständnis gibt es für jeden Schaden genau eine (Haupt-)Ursache und einen dafür Verantwortlichen. Diese personenzentrierte Ursachenforschung kann die Entstehung eines Zwischenfalls unmöglich erklären. Um das multifaktorielle Ursachengefüge aufzudecken, muss die Fehleranalyse systematisch erfolgen $\left(\rightarrow\right.$ Tab. 3). ${ }^{8}$

Bei der Untersuchung kritischer Ereignisse geht es also nicht um das „wer“, sondern um das „was“ und „warum“. Nicht um den einen Fehler, sondern um alle beitragenden Ursachen. Diese Sichtweise wird jedoch durch einige Phänomene der Sozialpsychologie erschwert: ${ }^{8}$

- Resultate eigenen Handelns werden gern äußeren Einflüssen zugeschrieben, während man die Handlungen anderer allein in deren Verantwortung sieht (Fundamentaler Attributionsfehler). 
- In der Analyse des Zwischenfalls wird die ursächliche Ereigniskette klar. Man ist verleitet anzunehmen, dies hätte allen Beteiligten zum Zeitpunkt des Zwischenfalls ebenso klar sein müssen (Rückschaufehler).

- Einer Person wird die Verantwortung zugeschoben, weil der Zwischenfall nicht eingetreten wäre, wenn sie anders gehandelt hätte. Andere Ursachen werden nicht bedacht (Kontrafaktischer Trugschluss).

- Die Auffassung von Handlungen als Ausdruck und Ergebnis des freien Willens führt dazu, äußere Umstände als Ursachen zu übersehen. ${ }^{7}$

Es muss bewusst erforscht werden, welche Informationen den Handelnden zur Verfügung standen und unter welchen Bedingungen sie arbeiteten; welche Ziele sie hatten, wie sie ihre Entscheidungen trafen und warum diese korrekt wirkten. Nur so kann die gesamte Fehlerkette aufgedeckt werden, um einer Wiederholung der Geschehnisse in Zukunft vorzubeugen.

\section{Die Fehlerkultur}

Die Wiener Qualitätsmanagerin Margit Wiederschwinger definierte Fehlerkultur als „Leitvorstellungen und Werte, die die Art und Weise bestimmen, wie in einem Unternehmen mit Fehlern umgegangen wird“. Zur Analyse einer Fehlerkultur hat die Management-Trainerin Elke M. Schüttelkopf ein Modell formuliert, das eine differenzierte Betrachtung von Strukturen und Inhalten erleichtert ( $\rightarrow$ Tab. 4 und 5). ${ }^{10}$ Anhand dieses Modells soll beispielhaft die im Gesundheitswesen und auch der Intensivpflege vorherrschende Fehlerkultur beschrieben werden. Individuelle Ausprägungen variieren zwischen Personen ebenso wie zwischen Stationen und Kliniken. Sie müssen daher auch individuell analysiert werden.

\section{Normen und Werte aktueller Fehlerkulturen}

Die Dynamik von Öffentlichkeit und Medien, durch die folgenreiche Behandlungs- und Pflegefehler den Charakter von Skandalen fern jeder Fassbarkeit erhalten, schlägt bis in die intraorganisationale Fehlerkultur durch. Patientensicherheit wird

Tab. 4 Modell der Fehlerkultur: Struktur - drei Säulen ${ }^{10}$

\begin{tabular}{|l|l|l|}
\hline Normen und Werte & Kompetenzen & Instrumente und Methoden \\
\hline $\begin{array}{l}\text { Richtungsweisend für } \\
\begin{array}{l}\text { Umgang mit Fehlern, Fehler- } \\
\text { risiken, Fehlerfolgen }\end{array}\end{array}$ & $\begin{array}{l}\text { Fähigkeit, Fehler zu ak- } \\
\text { zeptieren, thematisieren, } \\
\text { analysieren und mit ihren } \\
\text { emotionalen Auswirkungen } \\
\text { umzugehen }\end{array}$ & $\begin{array}{l}\text { Zutzung und Vermeidung } \\
\text { von Fehlern }\end{array}$ \\
\hline
\end{tabular}

Tab. 5 Modell der Fehlerkultur: Inhalte - vier Dimensionen ${ }^{10}$

\section{Vertrauen}

Bedingung für Kritikfähigkeit und offene, respektvolle Kommunikation über Fehler und Sicherheit. Vertrauen heißt nicht: „es wird schon alles gut gehen“, sondern: „meine Kollegen werden mit meiner Kritik und meinen Fehlern professionell umgehen“!

\section{Fehlervermeidung}

Bestreben nach Vermeidung bedrohlicher Fehler: bei erprobten Prozessen nicht vom Goldstandard abweichen

\section{Fehlerfreundlichkeit}

Bereitschaft zum Lernen aus Fehlern, zu Kreativität und Innovation, zum Betreten und Erproben neuer Wege

\section{Entwicklung}

Streben nach Verbesserung und Anpassung an neue Anforderungen, Reflexions- und Dialogfähigkeit

zur Selbstverständlichkeit, der Zwischenfall zum Tabu. Glazinski und Wiedensohler urteilen treffend: „Der Umgang mit Fehlern hat sich hier in der Regel darauf beschränkt, nach einem Schuldigen für das aufgetretene Problem zu suchen, diesen namentlich $\mathrm{zu}$ benennen und abzustrafen. Verleugnen nach außen und bestrafen nach innen, so könnte man überspitzt den Umgang mit Fehlern in der Patientenversorgung umreißen. Im angloamerikanischen Sprachgebrauch wird diese Vorgehensweise treffend auch als ,culture of blame“ bezeichnet." 11

Diese Kultur der Schuldzuweisung („naming, blaming and shaming“7) verhindert die Aufarbeitung von Fehlern und motiviert zu deren Vertuschung. Sie zerrüttet das Vertrauen aller ineinander und führt zu Diffusion und Abschieben von Verantwortung.

Ferner hat der steigende Kostendruck auf die Leistungserbringer im Gesundheitswesen eine Neuordnung der normativen Unternehmensführung angestoßen: Das Streben nach Rentabilität wird, ange- sichts wirtschaftlicher Zwänge, zur Maxime. Die Patientensicherheit mag weiterhin auf allen Fahnen stehen, wird jedoch gerade bei kostspieligen Entscheidungen verhandelbar.

Weitere Problematik rührt von hierarchischen Strukturen her. Im ärztlichen Bereich althergebracht, wird sie von Glazinski und Wiedensohler als Hindernis für konstruktive Fehlerkultur beschrieben: „Eine traditionell hierarchische ärztliche Führungsstruktur trägt leider nicht zur Förderung des Kritikvermögens gegenüber der Qualität der eigenen Arbeit bei, sondern leistet einer Haltung Vorschub, die Fehler ausschließlich als Makel begreift."11

Der zunehmende Mangel an Fachpersonal in der Pflege führt zu einem Erfahrungsgefälle innerhalb des Pflegeteams ${ }^{12}$, welches ähnliche Konsequenzen hervorbringt: Intra- und interdisziplinär ist das Äußern von Bedenken sowie das Ansprechen von Fehlern problematisch, selbst wenn Nachteile oder Gefahr für Patienten offensichtlich sind. Die amerikanische 


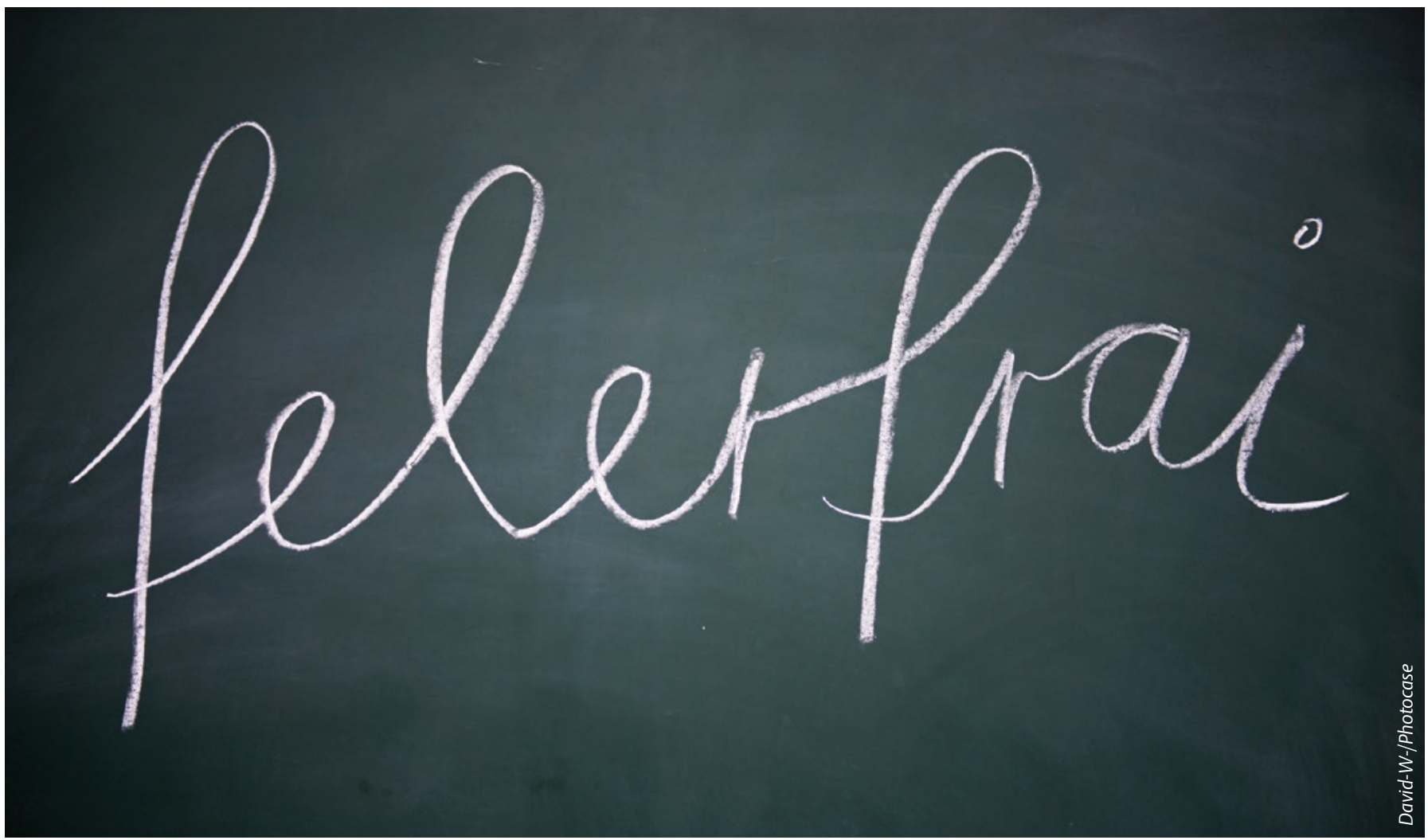

Studie „The Silent Treatment“ zeigte eindrucksvoll die fatalen Auswirkungen solcher Kommunikationsbarrieren. ${ }^{13}$

\section{Kompetenzen aktueller Fehlerkulturen}

Der konstruktive Umgang mit Fehlern setzt die Fähigkeit und Bereitschaft zum Lernen aus selbigen voraus. Lernwille und Lernreife von Individuen und Organisationen variieren stark. Auf dem normativen Fundament einer Null-Fehler-Mentalität entwickelt sich die Bereitschaft zum Lernen aus Fehlern eher schlecht.

Auch das Äußern und Annehmen konstruktiver Kritik sind bei der Beschäftigung mit Fehlern unentbehrlich. Die Entwicklung dieser Schlüsselqualifikationen hat in der Ausbildung von Pflegepersonen jedoch nur am Rande Platz.

Unerwünschte Ereignisse schaden nicht nur den Patienten - dem „Schuldigen“ erwachsen hohe psychosoziale Belastungen durch Vorwürfe, Ansehensverlust und Sanktionen. ${ }^{14}$ Weder Ausbildung noch das Arbeitsleben bereiten darauf vor. Da der mutmaßliche Verursacher nach klassischem Verständnis selbst schuld ist, hält sich die Unterstützung des Arbeitgebers bei der Bewältigung dieser Belastungen in engen Grenzen. Explizite Bewältigungsstrategien wie die kollegiale Ersthilfe finden sich nur vereinzelt und ohne organisierte Förderung.

\section{Instrumente und Methoden aktueller Fehlerkulturen}

Hier weist die Fehlerkultur in statu quo wohl die geringsten strukturellen Defizite auf. Anders als Normen, Werte und Kompetenzen sind Instrumente einfach $\mathrm{zu}$ implementieren. So können Instrumente wie Checklisten, das Crisis Resource Management (CRM), Berichtsysteme wie CIRS (Critical Incident Reporting System) und Analysetechniken wie die Root Cause Analysis aus höher entwickelten Fehlerkulturen entlehnt werden, etwa der der kommerziellen Luftfahrt. Auch Team- und Fallbesprechungen stellen Instrumente der Fehlerkultur dar.

Problematisch ist die eingeschränkte Nutzung dieser Instrumente. Mangelndes Fehler- und Sicherheitsbewusstsein auf allen Ebenen reduziert die Motivation hierzu. Auch die hohe und zunehmende Ar- beitsdichte in der Intensivpflege dämpft die Bereitschaft, sich zeitaufwendig mit Fehlern zu beschäftigen. Hinzu kommt das konsequente Fehlen von Belohnungsstrukturen für den konstruktiven Umgang mit Fehlern. Die organisationale Reaktion auf individuelle Fehler erschöpft sich meist in Sanktionen, wobei diese sich eher nach dem Schadensausmaß richten als nach tatsächlichem Verschulden. Die Tatsache, dass Fehler zudem nicht nach ihrer Entstehung, sondern nach ihrer Meldung an Vorgesetzte sanktioniert werden, leistet der Tendenz zur Vertuschung Vorschub.

\section{Inhaltliche Schwächen aktueller Fehlerkulturen}

Schüttelkopf hat der Dimension Vertrauen fundamentale Bedeutung eingeräumt. ${ }^{10}$ Leider wird gerade das Vertrauen durch die Mängel bei Normen und Werten stark beeinträchtigt. Führungskräfte verlieren das Vertrauen in Mitarbeiter, die Fehler machen. Umgekehrt reduzieren Sanktionen das Vertrauen der Pflegepersonen zu Vorgesetzten, mit Fehlern konstruktiv umgehen zu können. Die Angst davor, in der gegenwärtigen „Culture of blame“ eines 
Fehlers überführt zu werden, schafft Misstrauen unter Kollegen. Wenn dieser Fall eintritt, ist auch das Selbstvertrauen dahin.

Kaum besser sieht es mit der Fehlerfreundlichkeit und Entwicklung aus. Wer Fehler nicht wahrhaben will, wird nicht aus ihnen lernen. Höchstens dann, wenn ein Schaden auftritt. Der besondere Wert von Beinahe-Schäden als kostenlose Lektionen geht angesichts dieser Fehlerfeindlichkeit verloren.

Die Fehlervermeidung ist stark ausgeprägt, allerdings beschränkt durch den geringen Reifegrad des Umgangs mit Fehlern. Würde man sich nicht reaktiv, sondern präventiv mit Fehlern beschäftigen, könnte man die Fehlervermeidung wesentlich erfolgreicher gestalten. 5,7

\section{Normen und Werte einer idealen Fehlerkultur}

Das erste Erfordernis einer konstruktiven Fehler- und generativen Sicherheitskultur ist die Klarstellung der organisationalen und individuellen Ziele. Für jede Entscheidung, jeden Arbeitsprozess, jede einzelne Handlung muss die Sicherheit der Patienten oberste Priorität haben. Dieser Anspruch reicht weit über die Grenzen der Intensivstation hinaus und stellt sich auch an Management, Kostenträger und Politik. Die bloße Forderung nach Patientensicherheit ist nutzlos in Anbetracht der Tatsache, dass das Generieren von Sicherheit ständige Bemühung erfordert. Es müssen also auch die entsprechenden Möglichkeiten geschaffen und Mittel bereitgestellt werden. Entscheidungen auf allen genannten Ebenen müssen zeigen, dass Sicherheit eine höhere Priorität als Wirtschaftlichkeit hat. ${ }^{15}$ Hiermit einhergehen muss die Akzeptanz von menschlichen Fehlern als Tatsache. Nur wer sich bewusst macht, dass Menschen Fehler machen, kann sich produktiv mit ihnen beschäftigen. Dies gilt für die individuelle Pflegeperson ebenso wie für das gesamte Team, alle Führungsebenen und die Politik.

Schließlich sind Respekt und Wertschätzung für eine funktionierende Fehlerkultur von essenzieller Bedeutung. Nur wer sich gegenseitig wertschätzt und respektvoll behandelt, kann ohne Reibungsverluste über Fehler kommunizieren. „The Silent Treatment“ zeigte, wie respektloses
Verhalten Bemühungen für die Patientensicherheit unterminieren kann. ${ }^{13}$

\section{Die ideale Fehlerkompetenz}

In einer optimalen Fehlerkultur wird ständig über Sicherheit und Fehler kommuniziert: ${ }^{15}$ rege und beharrlich, offen und vertrauensvoll, wertschätzend und respektvoll. Der Fehlervermeidung dient das gegenseitige Hinweisen auf Fehler und Probleme. Diese Kritik wird konstruktiv, wertneutral und haltungserhaltend geäußert. Gleichzeitig wird sie respektvoll und dankbar angenommen. Im Zwischenfall wird gemeinsam Sorge getragen, dass der Schaden so gering wie möglich ausfällt. Generell wird es befürwortet, auch als erfahrene Pflegeperson Rat zu suchen oder um Hilfe zu bitten. Diese Hilfe wird auch gewährt, ohne vorschnelles Urteil über tatsächliche oder vermeintliche Fehler.

All dies kann erlernt und trainiert werden. Es existieren vielfältige Angebote von Kommunikationsseminaren und Anleitungen zu konstruktiver Kritik sowie spezielle Human-Factors- und Crisis Resource Management-Trainings. An dieser Stelle sei die Vorbildfunktion leitender und erfahrener Pflegepersonen hervorgehoben, die bei der Verbreitung dieser Kompetenzen als Multiplikator dienen können.

Die Fähigkeit zur systematischen Analyse von Zwischenfällen ist Ausgangspunkt der Lernprozesse. Von ihr hängt nicht nur ab, ob die am Zwischenfall beteiligten Personen zu Recht oder zu Unrecht sanktioniert werden, sondern auch, ob in Zukunft die richtigen Strategien zur Fehlervermeidung zur Anwendung kommen.

\section{Instrumente einer idealen Fehlerkultur}

Sanktionen müssen gut überlegt und differenziert eingesetzt werden. Die konsequente Bestrafung von vorsätzlichen Regelverstößen ist sinnvoll und notwendig. Bei echten Fehlern hingegen führt sie nicht zur Verbesserung der Patientensicherheit, im Gegenteil: Mitarbeiter, die ihre Bestrafung als ungerecht empfinden, verlieren das Vertrauen in die organisationale Fehlerkultur und halten sich zukünftig mit der Meldung von Fehlern und Zwischenfällen zurück. ${ }^{5}$ Die Motivation zu solchen Meldungen ist existenziell für eine konst- ruktive Fehlerkultur und darf durch Sanktionen nicht gefährdet werden. Ein noch mächtigeres Instrument ist die Belohnung. Sie verstärkt die Motivation, die die Triebfeder aller fehlerkulturellen Prozesse im Team der Intensivstation darstellt. Teamund Fallbesprechungen sowie Berichtsysteme wie CIRS sind hinreichend bekannte Instrumente. Es ist ihre ausgiebige Nutzung, die eine konstruktive Fehlerkultur ausmacht.

\section{Inhaltliche Dimensionen einer idealen Fehlerkultur}

In einer idealen Fehlerkultur gibt es kein Zuviel an Vertrauen. Niemand scheut sich, eigene Fehler zu melden oder um Rat zu fragen. Die Bildung von Vertrauen zum Selbst, zu anderen Pflegenden, anderen Berufsgruppen und Vorgesetzten ist Bedingung, dass die Fehlerkultur auf der Intensivstation ihre Möglichkeiten entfalten kann. Vertrauensfördernd wirkt ein respektvoller und wertschätzender Umgang miteinander. Auch gemeinsame Freizeitaktivitäten und Trainings (Kommunikations-, CRM-, Simulatortraining) beeinflussen Selbstvertrauen und Teamgeist positiv.

Die Fehlervermeidung einer idealen Fehlerkultur hat Systemcharakter; jeder Zwischenfall wird analysiert und daraus gewonnene Informationen zur Vermeidung seiner Wiederholung genutzt. Dabei wird Wert auf tiefgreifende Problemlösungen gelegt. Hierdurch bedingte Verzögerungen der Arbeitsabläufe werden nicht nur in Kauf genommen, sondern honoriert. $^{7}$

Für die Intensivpflege haben Fehlerfreundlichkeit und Entwicklung weniger Bedeutung als für kreative Branchen. Fehler als Lernchance wahrzunehmen, ist jedoch unerlässlich. Gleiches gilt für das permanente Bestreben, die Prozesse der Patientenversorgung sowie deren Standards an neue Erkenntnisse und Erfordernisse anzupassen. Als Instrument der Fehlervermeidung sind Standards und Standardprozeduren zweifellos wertvoll. Stures Festhalten an ihnen jedoch steht der hinterfragenden Grundhaltung konträr gegenüber, der in der modernen Pflegeausbildung wie auch in Human Factors- und CRM-Trainings hohe Bedeutung beigemessen wird. 
Fehlerkultur ändern: gern, aber wie? Die tiefgreifende Veränderung der Kultur eines Teams ist eine sensible Angelegenheit. Allzu forsche Versuche können zerrüttende Wirkung auf das Vertrauen haben. Nicht umsonst gibt es einen eigenen Führungsbereich nur für intraorganisationale Veränderungen: das Change Management.

- Langsamer ist schneller: Veränderung braucht Zeit. Große Eile verursacht Gegenwind.

- Transparenz: Nur wenn allen Beteiligten die Notwendigkeit, die Ziele und Planung der Veränderungen klar sind, können sie konstruktiv daran mitarbeiten.

- Partizipation: Es geht um das Team dann muss es sich auch einbringen dürfen. Ideen und Einwände berücksichtigen!

- Vorbilder und Belohnung: Das Lernen von Modellen spielt bei Werten und Verhaltensweisen eine große Rolle. Belohnung der gewünschten Entwicklung schafft die nötige positive Motivation.

- Widerstände konstruktiv nutzen: Widerstand offenbart Bedürfnisse. Etwa einen Mangel an Information über den Veränderungsprozess (Transparenz!) oder fehlende Identifikation mit diesem (Partizipation!). Widerstand kann auch auf Schwächen im Veränderungsmanagement hinweisen.

O FAZIT

Konstruktive Fehlerkultur beginnt im Kopf - mit dem Wissen, dass Menschen Fehler machen, und dem Verständnis für die Notwendigkeit, sich mit diesen auseinanderzusetzen. Das Verständnis der Fehlerentstehung ist entscheidend für die korrekte Behandlung: Sanktionen wirken nur bei vorsätzlichen Regelverstößen.

Das Auftreten von Aufmerksamkeits- und Gedächtnisfehlern wird durch Strafe nicht beeinflusst. Das Arbeitsumfeld muss so angepasst werden, dass es die Wahrscheinlichkeit ihrer Entstehung reduziert und Fehlerdetektion sowie -korrektur erleichtert. Die personenzentrierte Fehleranalyse ist zugunsten der systematischen aufzugeben. Fast immer tragen latente Bedingungen zu Zwischenfällen bei, die sich dem Einfluss der einzelnen Pflege- person entziehen. Die Frage „wer?“ ist bei der Ursachenforschung nicht zielführend. Sinnvoller ist die Frage, was jeder Einzelne tun kann, um einer Wiederholung des Geschehenen vorzubeugen. Im Team der Intensivstation kommt es darauf an, das Vertrauen zu stärken und einen respektvollen, wertschätzenden Umgang zu pflegen. Es sollte erwünscht sein, sich gegenseitig um Rat zu fragen und auf Fehler und Probleme hinzuweisen. Fall- und Teambesprechungen, Supervisionen und gemeinsame CRM-Trainings sind probate Mittel zur Steigerung der Fehlerkompetenz im Team. Ferner muss die Arbeitsdichte in der Intensivpflege reduziert werden. Für die Auseinandersetzung mit Fehlern bleibt keine Zeit, wenn die Patientenversorgung an sich schon zu kurz kommt. ${ }^{12}$ Politik, Kostenträger und Management müssen Möglichkeiten schaffen, die Beschäftigung mit Fehlern in die Arbeitsprozesse der Intensivpflege zu integrieren. Die Entwicklung fehlerkultureller Kompetenzen muss stärker in der Ausbildung, in Weiterbildungen und in der Einarbeitung neuer Mitarbeiter verankert werden. Für die Patientensicherheit ist ein Wandel der Fehlerkultur notwendige Bedingung. $\mathrm{Er}$ kann jedoch nur erfolgreich sein, wenn sich alle Ebenen des Gesundheitswesens daran beteiligen, wenn aus der Forderung nach weniger Fehlern die Frage wird: Was können wir zu einer konstruktiven Fehlerkultur beitragen?

\section{Literatur}

1 Aktionsbündnis Patientensicherheit: Glossar zur Patientensicherheit. Online unter www.aps-ev. de/patientensicherheit/glossar; letzter Zugriff: 28.07.2015

2 Valentin A, Capuzzo M, Guidet B, Moreno R, Dolanski L, Bauer P, Metnitz P. Patient safety in intensive care: results from the multinational Sentinel Events Evaluation (SEE) study. Intensive Care Medicine 2006, 32: 1591

3 Valentin A, Capuzzo M, Guidet B, Moreno R, Metnitz B, Bauer P, Metnitz P. Errors in administration of parenteral drugs in intensive care units: multinational prospective study. British Medical Journal 2009, 338: b814

4 Amalberti R. The paradoxes of almost totally safe transportation systems. Safety Science 2001, 37: 109

5 Rall M, Manser T, Guggenberger H, Gaba D, Unertl K. Patientensicherheit und Fehler in der Me- dizin. Anästhesiologie Intensivmedizin Notfallmedizin Schmerztherapie 2001, 36: 321

6 Weingardt M. Fehler zeichnen uns aus: transdisziplinäre Grundlagen zur Theorie und Produktivität des Fehlers in Schule und Arbeitswelt. Bad Heilbrunn: Julius Klinkhardt; 2004

7 Reason JT. Human Error: models and management. The British Medical Journal 2000, 320: 768

8 Hofinger G. Fehler und Unfälle. In: BadkeSchaub P, Hofinger G, Lauche K. Human Factors. Berlin: Springer; 2012

9 Wiederschwinger M. Fehlermanagement als Chance. Konstruktive Fehlerkultur als integraler Bestandteil der Qualitätsarbeit. Wien: Vortrag am 16.04.2004

10 Schüttelkopf EM. Erfolgsstrategie Fehlerkultur. In: Ebner G, Heimerl P, Schüttelkopf EM. Fehler - Lernen - Unternehmen. Frankfurt a.M.: Peter Lang Internationaler Verlag der Wissenschaften; 2008

11 Glazinski R, Wiedensohler R. Patientensicherheit und Fehlerkultur im Gesundheitswesen: Fehlermanagement als interdisziplinäre Aufgabe im Gesundheitswesen. Eschborn: Brainwave Wissenschaftsverlag; 2004

12 Isfort M, Weidner F, Gehlen D. Arbeitsverdichtung zeigt gefährliche Folgen. PflegenIntensiv 2012, 3: 1

13 Maxfield D, Grenny J, Lavandero R, Groah L. Silent kills. intensiv 2012, 20: 126

14 Gutachten 2003 des Sachverständigenrates für die Konzertierte Aktion im Gesundheitswesen. Online unter http://dip21.bundestag.de/ dip21/btd/15/005/1500530.pdf, letzter Zugriff: 28.07.2015

15 Reason JT. Managing the risks of organizational accidents. Aldershot: Ashgate; 1997

\section{E AUTOR}

Christian Bibo

Fach-Gesundheits- und Krankenpfleger für Intensivpflege, Praxisanleiter, Universitätsmedizin der Johannes Gutenberg-Universität Mainz, Weiterbildung in den Gesundheitsfachberufen E-Mail: christian.bibo@unimedizin-mainz.de

三 BIBLIOGRAFIE

DOI 10.1055/s-0041-105918

Intensiv 2015; 23 (6): 321-327

(c) Georg Thieme Verlag KG

Stuttgart · New York · ISSN 0942-6035 